\title{
ETHANOL IN FATAL ROAD TRAFFIC ACCIDENTS. THE PREVALENCE OF ETHANOL IN FATAL ROAD TRAFFIC ACCIDENTS IN THE BAHAMAS: THE NEED FOR A CONTROLLED STUDY.
}

Danny Davis, Veronica Ferguson, James Carey and Elburt Ferguson

School of Natural Sciences and Environmental Studies, College of The Bahamas, Forensic Science Services, Royal Bahamas Police Force

https://doi.org/10.15362/ijbs.v13i0.59

\section{ABSTRACT}

There are no documented studies of the role of ethanol in fatal road traffic accidents in The Bahamas. This paper provides a retrospective analysis of the involvement of ethanol in road traffic fatalities for 2000-2002 in The Bahamas. In the three-year period covered by this study 185 traffic fatalities were recorded, post mortem blood samples from 139 decedents were analyzed for blood alcohol concentration (BAC). The involvement of ethanol in many of these fatalities was not conclusive because BAC at the time of the accident cannot be confidently extrapolated from post mortem BAC data. In an effort to understand the involvement of ethanol in road traffic accidents we propose that BAC of all participants in road traffic accidents involving injuries should be recorded. This proposal is based on, and supported by, the analyses of the available data. Analysis of the data highlights its deficiencies and that of the data collection system. The case for a systematic study is presented.

Recent civic and governmental campaigns have endeavored to raise the collective awareness of the Bahamian public to the perils of drinking and driving. The success, or failure, of such programs cannot be ascertained unless the involvement of ethanol in fatal traffic accidents is quantified. The involvement of ethanol in fatal traffic accidents or accidents involving injuries is not known in this population. In countries that compile data on ethanol related motor vehicle accidents, the number of ethanol related traffic fatalities is often quoted as a percentage of the total number of traffic fatalities in a given year (NHTSA, 2004). These values are often used as measures of the success of "anti-drinking and driving" campaigns and are invaluable for sustaining interest and funding for these campaigns. Currently, Bahamian law allows for the prosecution of a person for "Driving a motor vehicle while under the influence of alcohol 
or drugs" ("The Road Traffic Act," 2001) but no provision exists for mandatory testing of all occupants of all vehicles involved in fatal traffic accidents. The legal limit for blood alcohol concentration is $80 \mathrm{mg} / 100 \mathrm{~mL}$. Roadside breath alcohol analyzers (Breathalyzers ${ }^{\circledR}$ ) are not used in The Bahamas to determine breath alcohol concentration therefore our discussion will be restricted to blood alcohol concentration.

In an effort to understand the involvement of ethanol in fatal traffic accidents all traffic fatalities from 2000 to 2002 inclusive, were reviewed. In The Bahamas a 1-year limit between the accident and death is imposed for the classification of fatalities as being "related to a traffic accident"("The Penal Code," 2001). The Road Traffic Division of the Royal Bahamas Police Force is responsible for the classification of a death as being "related to a traffic accident". This classification is based on the pathologist's report. The National Highway Traffic Safety Administration in the United States (our nearest jurisdiction) restricts the classification of "traffic fatalities" to deaths that occur within 30 days of the traffic accident (CDC, 2001).

While considerable data exist on the blood alcohol concentration (BAC) for drivers and occupants of vehicles in many major cities around the world (Logan \& Schwilke, 1996) no data is published as it relates to the BAC of persons involved in road traffic fatalities in The Bahamas. A retrospective analysis of the road traffic accident data and forensic analyses of stored blood samples provided a data set of 185 cases for the 3-year period of this study. Analysis of the data allow us to guide policy makers as to how changes can be made which will permit systematic study of the role of ethanol in fatal road traffic accidents.

\section{METHOD}

Post mortem blood samples were collected during autopsy and submitted to the Royal Bahamas Police Force, Forensic Science Services (Nassau, Bahamas) for analysis. Blood ethanol content was determined using a gas chromatograph (Perkin Elmer Autosystem XL) fitted with a headspace sampler (Perkin Elmer, HS-40XL) and a flame ionization detector. The chromatographic conditions are described by Brown and Long (Brown \& Long, 1988), with the following modifications; detector temperature $(150 \infty \mathrm{C})$, cycle time (11 min.), and carrier flow rate $(7.5 \mathrm{~mL} / \mathrm{min})$. All reagents were purchased from Aldrich Chemicals (Milwaukee, Wisconsin) and used without further purification. Data were analyzed using commercial software (Minitab version 13.1, Minitab Corporation, Pennsylvania, USA).

\section{RESULTS}

A total of 185 traffic fatalities were recorded for the three-year period 2000 to 2002. The BAC was undetermined for 46 (25\%) of the decedents while 38 (21\%) of the recorded BAC values were greater than $80 \mathrm{mg} / 100 \mathrm{~mL}$ of blood. The remaining 101 (73\%) samples had BAC values that were below the $80 \mathrm{mg} / 100 \mathrm{~mL}$ of blood limit. A summary of the missing data is provided as Table 1. A comprehensive analysis of some aspects of the data is not possible due to the number of missing data points. 
Table 1: Summary of missing data in the 2000-2002 data set for fatal traffic accidents in The Bahamas

\begin{tabular}{llccccccc}
\hline & Number & \multicolumn{7}{c}{ Missing Data Attribute } \\
\cline { 2 - 8 } Year & $\begin{array}{l}\text { of } \\
\text { accidents }\end{array}$ & $\begin{array}{l}\text { Time of } \\
\text { accident }\end{array}$ & $\begin{array}{l}\text { Date of } \\
\text { death }\end{array}$ & $\begin{array}{l}\text { Time of } \\
\text { death }\end{array}$ & $\begin{array}{l}\text { Hospital } \\
\text { stay }\end{array}$ & $\begin{array}{l}\text { Type of } \\
\text { accident }\end{array}$ & $\begin{array}{l}\text { BAC } \\
\text { analysis }\end{array}$ & Age \\
\hline 2000 & 75 & 6 & 21 & 25 & 21 & 6 & 22 & 3 \\
2001 & 56 & 0 & 1 & 2 & 1 & 0 & 13 & 0 \\
2002 & 54 & 0 & 0 & 11 & 2 & 0 & 11 & 2 \\
\hline Total & 185 & 6 & 22 & 38 & 24 & 6 & 46 & 5 \\
\hline
\end{tabular}

The duration of hospitalization was calculated from the difference between the time of the accident and the time of death. The date of death is not known for $28 \%$ of the fatalities that occurred as a result of traffic accidents in 2000 whereas in 2001 and 2002 the number of missing entries is reduced to $2 \%$ and $0 \%$, respectively.

The distribution of BAC as a function of the pathway to death, i.e., was the decedent hospitalized prior to death or was the decedent pronounced dead on arrival (DOA) at the hospital, is shown in Figure 1.

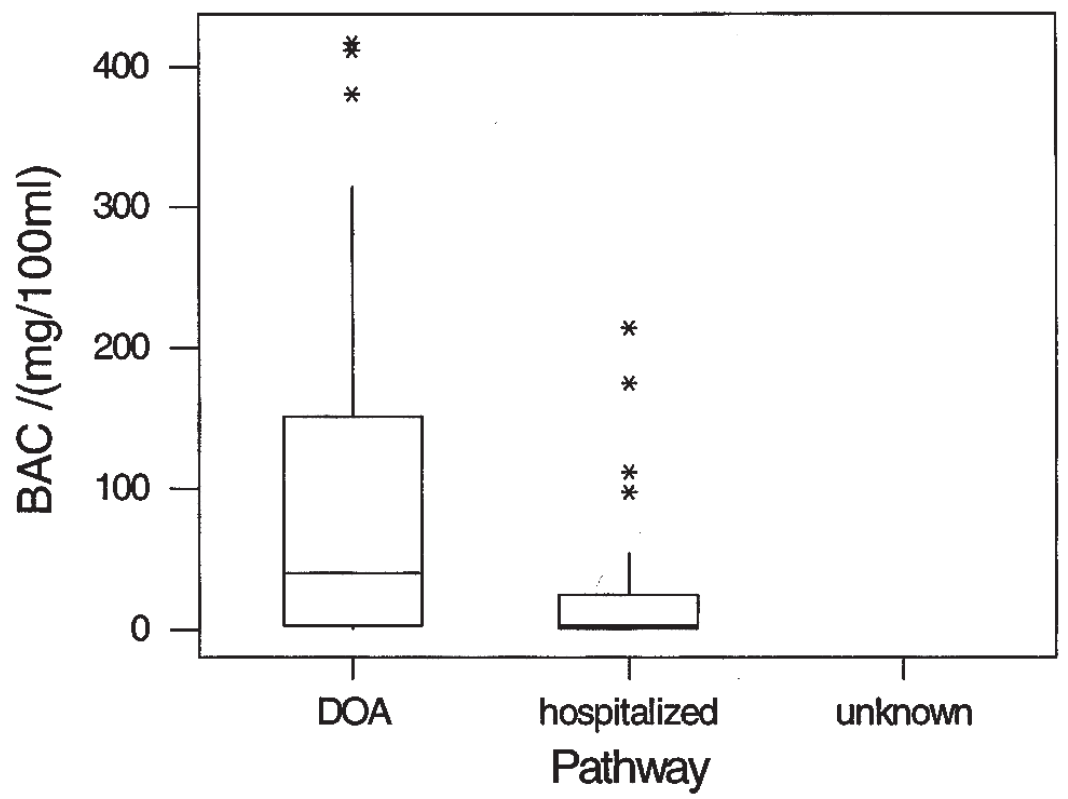

Figure 1: Boxplot of distribution of blood alcohol concentration (BAC) for hospitalized decedents and victims that were pronounced "dead on arrival" (DOA) at the hospital.

Accidents are defined as either multiple vehicular accidents or singular vehicular accidents. All accidents involving pedestrians, bicyclists or more than one motor vehicle were classified as multiple vehicular accidents. Singular vehicular accidents are classified as those accidents involving a single motor 
vehicle and a fixed object, e.g., a utility pole. The type of accident was known for $88 \%$ of the recorded fatal traffic accidents in 2000. The data sets for 2001 and 2002 are complete in regard to the accident type. The distribution of BAC as a function of type of accident type is shown in Figure 2. The distribution of BAC of DOA decedents and the distribution of BAC of hospitalized decedents as a function of accident type is shown in Figures 3 and 4, respectively.

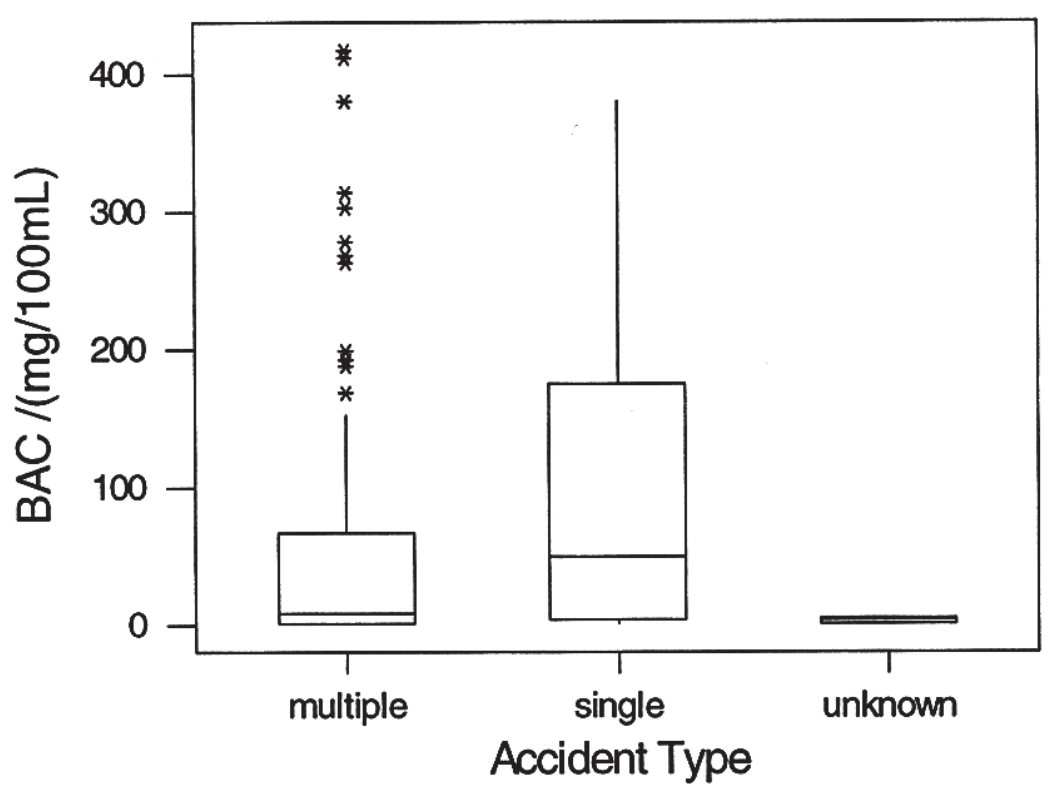

Figure 2: Boxplot of distribution of BAC based on accident type.

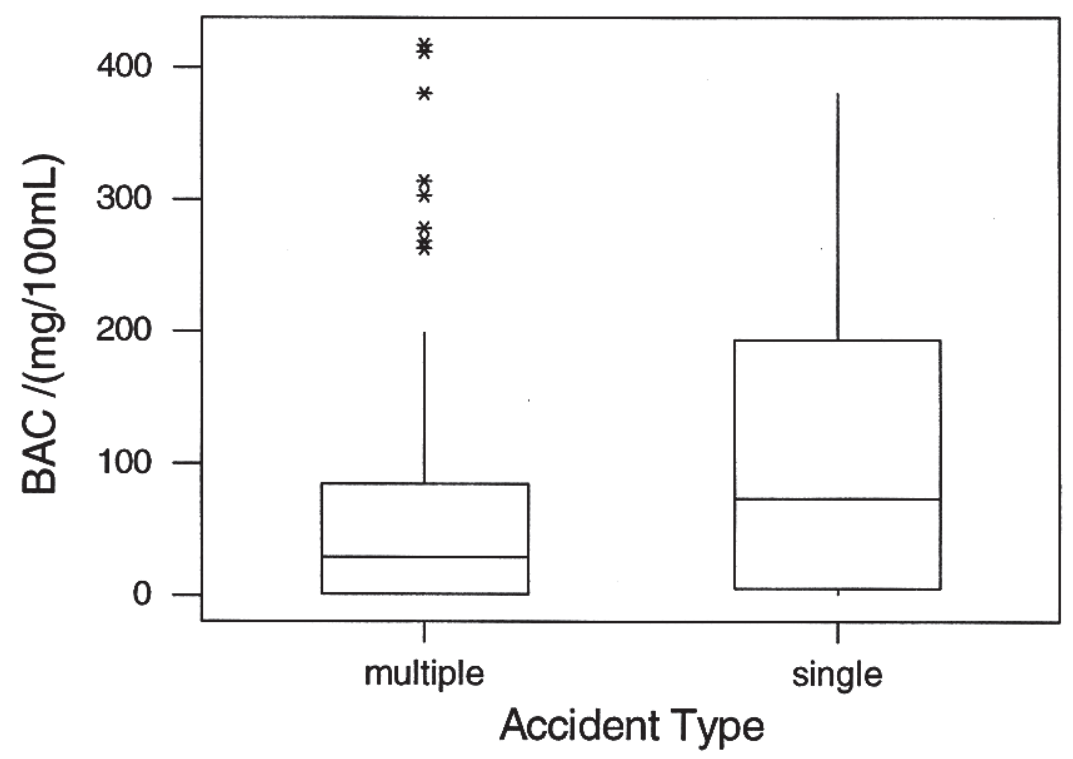

Figure 3: Boxplot of BAC distribution of DOA decedents by accident type. 


$$
\text { type. }
$$

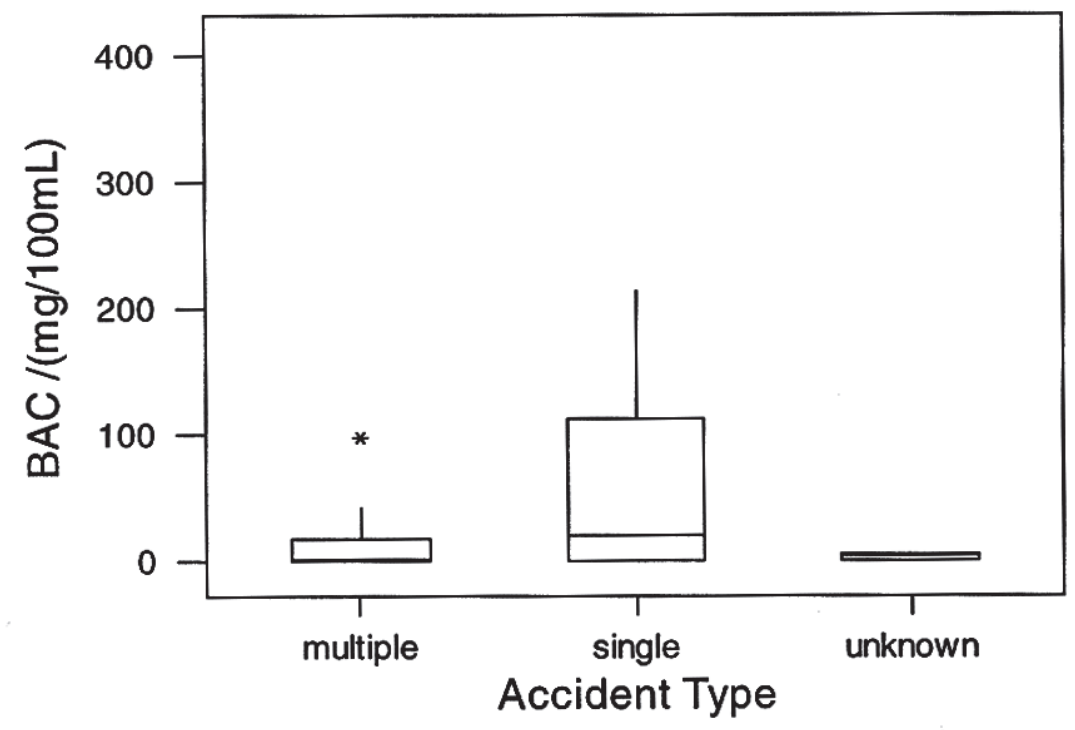

\section{DISCUSSION}

As is usually the case with a retrospective study (especially those involving fatalities) factors such as insufficient sample or access to the original donor prohibited completion of the decedent BAC data. The data set was not complete and we did not impose any general exclusion criteria. Due to the fact that the major source of error was preanalytical, the application of any exclusion criteria would have created subsets that would inherit the same preanalytical errors.

The rate of ethanol metabolism in a typical $70 \mathrm{~kg}$ person is 7-10 g/hour; therefore it is possible for a person to reduce his BAC to below a legally intoxicated level within a few hours (Todd, Sanford, Davidsohn, \& Henry, 1984). Hospitalization of any duration, i.e., the decedent was not pronounced dead on arrival (DOA) at the hospital, will result in a lower BAC in post mortem samples than the actual $\mathrm{BAC}$ at the time of the accident. The values that most closely approximate the accident BAC are those in which the victim succumbed to injuries at the scene. Therefore, the duration of hospitalization must be known in order to eliminate false negative indicators for the BAC at the time of the accident. If the victim was hospitalized it is expected that the post mortem BAC will be significantly lower than the true value at the time of the accident due to ethanol metabolism.

The current system of waiting until the hospitalized patient succumbs to his injury to obtain a BAC sample does not provide reliable results. The apparent wider distribution (Figure 1) for decedents who succumb to injuries at the scene and are pronounced dead on arrival (DOA) at the hospital in contrast to those who succumb after a period of hospitalization, supports the hypothesis that hospitalized decedents will have lower BAC values than DOA decedents. The distribution of BAC of singular vehicular accident decedents is wider 
than that of multiple vehicular accident decedents because of the possibility that the decedent in a multiple vehicular accident was not the person intoxicated at the time of the accident (Figure 2). Inherent in this conclusion is the assumption that the intoxicated driver was responsible for the accident; this assumption is not necessarily valid for all cases. The lower BAC distribution for multiple vehicular accidents (Figure 2) is not due to hospitalization as demonstrated in Figures 3 and 4. A shortcoming of the current system is that BAC analyses are not completed on all drivers involved in multiple vehicular accidents. The apparently narrower BAC distribution of DOA decedents (Figure 3) and hospitalized decedents (Figure 4) in multiple vehicular accidents in contrast to singular vehicular accident decedents suggest that the wider BAC distribution that was observed for singular vehicular accident decedents compared to multiple vehicular accidents decedents in Figure 2 cannot be attributed to ethanol metabolism during hospitalization. If ethanol metabolism were responsible for this apparent difference in distribution we would expect to see identical boxplots in Figure 4.

In 2000, six BAC values (8\%) are missing for multiple vehicular fatal accidents while BAC data are not available for $15 \%$ of the total single vehicular fatal accidents in the same year. In 2001 and 2002 the number of missing BAC values for multiple vehicular accidents decreased to $4 \%$ and $9 \%$, respectively. The number of multiple vehicular accidents was almost half that of singular vehicular fatal accidents in the three year period of this study. The fact that BAC results are not available for all singular vehicular accident victims is indicative of a breakdown in the current system. In the absence of a defined investigative policy there is variance in what samples should be taken, inevitably some samples will not be collected or analyzed.

It is not unreasonable to assume that in most instances fatal multiple vehicular accidents involving an automobile and a bicyclist will result in the death of the bicyclist and not the driver of the automobile. If the driver of the vehicle were driving under the influence of alcohol then the current testing protocol would not result in the driver being tested and the involvement of ethanol will be underestimated. The data suggest that all drivers in multiple vehicular accidents should be tested for BAC.

The reliability of the BAC value is dependent on factors not accounted for in this study, namely: (i) post mortem ethanol production (Briglia, Bidanset, \& Dal Cortivo, 1992; Canfield, Kupiec, \& Huffine, 1993) (ii) source of blood sample, i.e., heart, or peripheral blood vessel (Briglia et al., 1992) (iii) collection and storage of biological samples (Sreerma \& Hardin, 2003) and (iv) type of biological fluid, i.e., urine, blood, vitreous humor, etc. (Hardin, 2002). Common to all of the aforementioned factors is the fact that gross trauma, as could be expected in a traffic accident involving a fatality, is a significant factor in the value of the reported BAC (Winek, Winek, \& Wahba, 1995).

The large percentage of decedents with undetermined BAC makes it difficult to come to any conclusions regarding the role of alcohol in road traffic fatalities. The data clearly indicates that methods and systems must be introduced or modified to reduce, and ultimately eliminate, the number of undetermined BAC for road traffic fatalities in The Bahamas. 


\section{CONCLUSIONS}

The scope of the analysis of the current data is limited by the fact that the reported $\mathrm{BAC}$ values are not indicative of the $\mathrm{BAC}$ values at the time of the accident. The reduced distribution of the BAC of decedents in multiple vehicular accidents compared to BAC of decedents in single vehicular accidents supports the need to test all drivers involved in multiple vehicular accidents.

Reliable data on the involvement of ethanol in traffic accidents in The Bahamas can only be compiled if all occupants of all vehicles are tested for breath and blood alcohol content at the time of the accident. One must remain cognizant of the fact that the breath alcohol content is only used as an indicator of the BAC. This study shows that in order to make definitive statements regarding the role of ethanol in road traffic fatalities a systematic approach to data collection must be implemented. The preferred implementation should include determination of the BAC of all individuals in traffic accidents involving injuries; these samples should be collected within two hours or as soon as possible after the accident. Any campaign intended to reduce the occurrence of alcohol-related traffic fatalities must target the offending subset of drivers. This subset can only be identified as a result of controlled and planned data collection and analysis. The medicolegal implications of our findings are significant regarding the revision of drinking and driving legislation.

\section{REFERENCES}

Administration, U. S. N. H. T. S. (2004). Traffic Safety Facts 2002 (No. HS 809 620). Washington: U.S. Department of Transportation, National Highway Traffic Safety Administration.

Briglia, E. J., Bidanset, J. H., \& Dal Cortivo, L. A. (1992). The Distribution of Ethanol in Postmortem Blood Specimens. Journal of Forensic Sciences, 37(4), 991-998.

Brown, D. J., \& Long, W. C. (1988). Quality Control in Blood Analysis: Simultaneous Quantitation and Confirmation. Journal of Analytical Toxicology, 12, 279-283.

Canfield, D. V., Kupiec, T., \& Huffine, E. (1993). Postmortem Alcohol Production in Fatal Aircraft Accidents. Journal of Forensic Sciences, 38(4), 914-917.

CDC. (2001). Alcohol Involvement in Fatal Motor-Vehicle Crashes - United States, 1999-2000. Morbidity and Mortality Weekly Report, 50(47), 10641065.

Hardin, G. G. (2002). Postmortem Blood and Vitreous Humor Ethanol Concentrations in a Victim of a Fatal Motor Vehicle Crash. Journal of Forensic Sciences, 47(2), 402-403.

Logan, B. K., \& Schwilke, E. W. (1996). Drug and Alcohol use in Fatally Injured Drivers in Washington State. Journal of Forensic Sciences, 41(3), 505-510.

The Penal Code, Statute Laws of The Bahamas, Chapter 84, Section 306 (2001). 
The Road Traffic Act, Statute Laws of The Bahamas, Chapter 220, Section 49, 49A-49E (2001).

Sreerma, L., \& Hardin, G. G. (2003). Improper Sealing Caused by the Styrofoam Integrity Seals in Leakproof Plastic Bottles Lead to Significant Loss of Ethanol in Frozen Evidentiary Urine Samples. Journal of Forensic Sciences, 48(3), 672-676.

Todd, J. C., Sanford, A. H., Davidsohn, I., \& Henry, J. B. (1984). Therapeutic Drug Monitoring and Toxicology. In Clinical diagnosis and management by laboratory methods (17th ed., pp. 1502). Philadelphia: Saunders.

Winek, C. L., Jr., Winek, C. L., \& Wahba, W. W. (1995). The Role of Truma in Postmortem Blood Alcohol Determination. Forensic Science International, 71, 1-8.

Danny Davis is a lecturer in chemistry at the College of The Bahamas (COB). He is an alumnus of COB (AA Chemistry '84); Acadia University, Nova Scotia, Canada (B.Sc.H. Chemistry '91); and Dalhousie University, Nova Scotia, Canada (Ph.D. Chemistry ‘96).

Veronica Ferguson joined the Royal Bahamas Police Force (RBPF) Forensic Science Laboratory in 1994. She is an alumnus of COB (AA Chemisty/ Biology '94) and Florida Atlantic University, Florida (B.Sc. Chemistry 97 and M.Sc. Chemistry ‘99).

Elburt Wesley Ferguson holds the rank of Assistant Superintendent of Police and currently serves as Deputy Director of the RBPF Forensic Science Laboratory. He is an alumnus of COB (AA Biology '79); Alma College, Michigan (B.Sc. Biology '83) and the University of Strathclyde, Scotland (M.Sc. Forensic Science '89)

James Carey earned his Bachelor of Science Degree in Chemistry and Biochemistry from the University of The West Indies in 1976 and his Masters of Science Degree in Forensic Science from the University of Strathclyde, Glassgow Scotland in 1979. He served as director of the RBPF Forensic Science Laboratory for 28 years. 